\title{
WITHANIA COAGULANS (STOCKS) DUNAL: BIOTECHNOLOGICAL ACHIEVEMENTS AND PERSPECTIVES
}

\author{
A review \\ Jaime A. TEIXEIRA da SILVA ${ }^{1 *}$, Mafatlal M. KHER ${ }^{2 * *}$, Deepak SONER ${ }^{2}$, M. NATARAJ ${ }^{2 * * *}$ \\ ${ }^{1}$ P. O. Box 7, Miki-cho post office, Ikenobe 3011-2, Kagawa-ken, 761-0799, Japan \\ ${ }^{2}$ B.R. Doshi School of Biosciences, Sardar Patel University \\ Sardar Patel Maidan, Vadtal Rd., P.O. Box 39, Vallabh Vidyanagar, Gujarat, 388120, India
}

Received: April 2015; Accepted: June 2015

\begin{abstract}
Withania coagulans (Stocks) Dunal is an important medicinal plant of the Solanaceae. Biotechnological studies on this plant started in 2009 and are still in a nascent phase of development. Even so, some important advances have been made, particularly in the field of tissue culture, which is an important means for its large-scale propagation and in vitro conservation. This review focuses on methods for surface sterilization, culture initiation, multiplication, rooting and acclimatization of W. coagulans.
\end{abstract}

Key words: adventitious shoot regeneration, in vitro rooting, micropropagation, tissue culture, RAPD, vegetable rennet, Withania coagulans

\section{INTRODUCTION}

Withania coagulans (Stocks) Dunal (Solanaceae), commonly known in English as Indian cheese maker, Indian rennet or vegetable rennet and with trade names panir (or paneer) doda, dodi, bandh and dhodi, is a medicinal herb whose biotechnological attributes have not been extensively explored, unlike better known W. somnifera. W. coagulans is a seed propagated, berry bearing bush that has ample medicinal properties conferred by the presence of many biologically active compounds including alkaloids, steroids, phenolic compounds, tannins, saponins, carbohydrates, proteins, amino acids, organic acids and withanolides (steroidal lactones). It is known that these bioactive compounds possess antihyperglycaemic, antihyperlipidemic, hypolipidemic, antiinflammatory, antimutagenic and anticancer/ anticarcinogenic, hepatoprotective, immunomodulatory, antifungal, antibacterial, antihelminthic, hypocholesterolemic, free radical scavenging, wound healing, cardiovascular, immunosuppressive and diuretic activities (reviewed in Gupta
2012; Jain et al. 2012; Gupta \& Keshari 2013; and references therein). Excessive collection of W. coagulans from wild resources has marked this plant species as threatened and endangered (Rawat 2008), although it is not listed on the IUCN Red List www.iucnredlist.org). The association between the roots of W. coagulans and arbuscular mycorrhizal fungi may confer upon the plant an ability to survive harsh climatic conditions such as drought (Panwar \& Tarafdar 2006).

The use of tissue culture and biotechnology would allow the mass production of medicinally important compounds such as the withanolides from rare germplasm. According to Mishra et al. (2013), the difference between $W$. somnifera and $W$. coagulans is that withanolides are produced primarily in the roots of the former and primarily in the aerial parts of the latter. For sampling natural populations in order to identify superior genotypes or to assemble diverse genotypes for future breeding programmes, a suitable sampling procedure is required (described by Gilani et al. 2009). In this study, seven populations of W. coagulans from diverse districts in Pakistan 
were analysed using PBA (P450 based analogue) markers, identifying low interpopulation but high intrapopulation diversity. The diversity can further be mined by nondestructive sampling of tissues and propagated by in vitro techniques as summarized in this minireview.

A micropropagation protocol typically consists of explant collection, surface sterilization, establishment of aseptic cultures, shoot induction and multiplication, rooting, acclimatization, screening of genetic fidelity and performance of micropropagated plants in field conditions. Nodal explants from fieldgrown plants or from in vitro seedlings are the most common explants for the tissue culture of $W$. coagulans (Table 1). There are only two alternative regeneration protocols for $W$. coagulans. One of them described adventitious shoot regeneration from leaf segments of field-grown plants (Jain et al. 2011), while the other described the use of leaves from axillary shoots emerging from nodes and shoot tips of $i n$ vitro-raised shoots (Mishra et al. 2013). There is also only one report on callus-mediated regeneration from leaves and internode segments of in vitro seedlings of W. coagulans (Valizadeh \& Valizadeh 2009). There are no reports that consider direct or callus-mediated somatic embryogenesis in W. coagulans. The literature (Table 1) indicates that Murashige and Skoog's (MS, Murashige \& Skoog 1962) medium has been the best choice for culture initiation, multiplication and rooting. No studies found any other basal media to be important. Most studies employed $N^{6}$-benzyladenine (BA) alone or in combination with kinetin $(\mathrm{Kn})$ for shoot induction and multiplication (Table 1). Only one report is available in which a cytokinin, meta topoline (mT), was found to be the most effective for axillary shoot multiplication from nodal explants (Joshi 2014). Joshi (2014) studied the effects of $0.5,1.0,1.50,2.0$ and $2.50 \mathrm{mg} \cdot \mathrm{l}^{-1}$ of $\mathrm{BA}, \mathrm{Kn}$, thidiazuron (TDZ), zeatin (Zea) and $\mathrm{mT}$ in combination with $50 \mathrm{mg} \cdot \mathrm{l}^{-1}$ adenine sulphate (AdS) and $0.1 \mathrm{mg} \cdot 1^{-1} \alpha$-naphthaleneacetic acid (NAA). The best shoot multiplication index $(79.17 \%)$ was achieved with MS medium supplemented with $2.50 \mathrm{mg} \cdot 1^{-1} \mathrm{TDZ}, 50 \mathrm{mg} \cdot \mathrm{l}^{-1} \mathrm{AdS}$ and $0.1 \mathrm{mg} \cdot \mathrm{l}^{-1} \mathrm{NAA}$ but all regenerated axillary shoots were hyperhydric (Fig. 1A). On the other hand, when the MS medium was supplemented with $2.50 \mathrm{mg} \cdot \mathrm{l}^{-1} \mathrm{mT}, 50 \mathrm{mg} \cdot \mathrm{l}^{-1}$ AdS and $0.1 \mathrm{mg} \cdot 1^{-1} \mathrm{NAA}$, the multiplication index of healthy shoots was $75 \%$ (Fig. 1B). Indole-3-acetic acid (IAA) or indole-3-butyric acid (IBA) was the most frequently used as auxins for inducing in vitro rooting, usually in the presence of half-strength MS medium (Table 1). Jain et al. $(2009,2011)$ reported that the addition of phloroglucinol (PG) and choline chloride (CC) to the basal medium enhanced the rooting of in vitro cultured W. coagulans shoots.
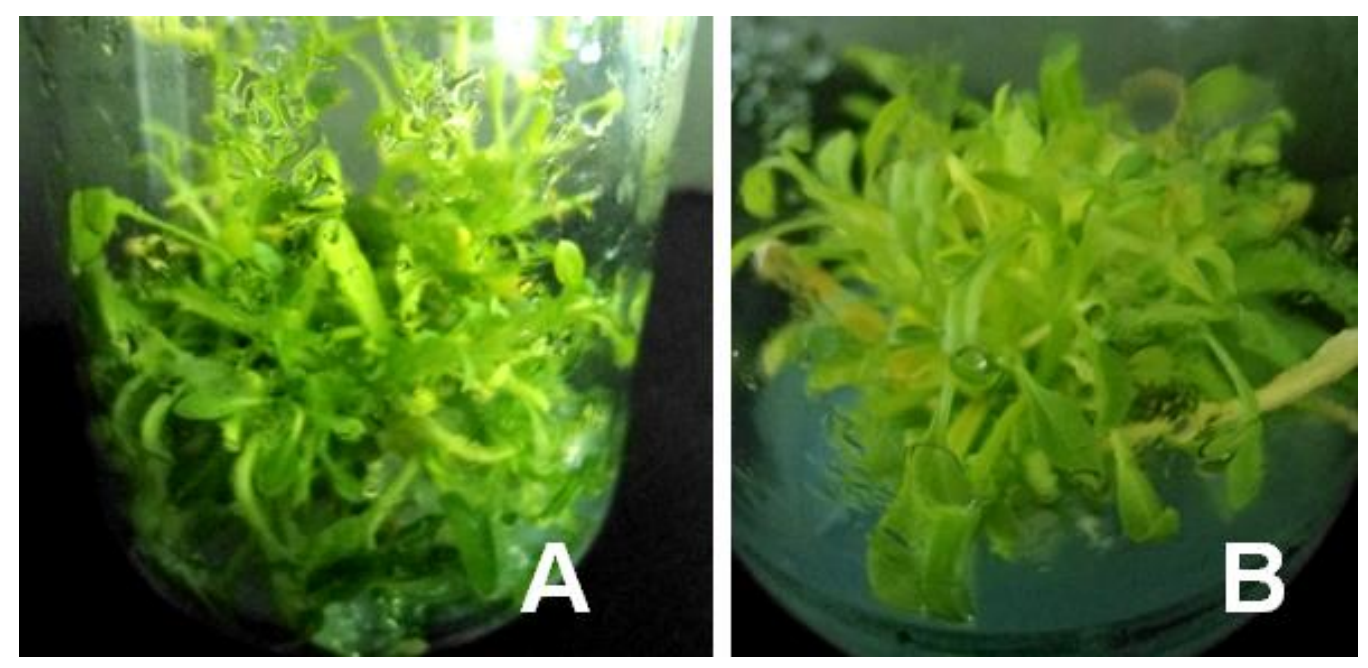

Fig. 1. Tissue culture of Withania coagulans (Stocks) Dunal (45-day-old cultures; unpublished results). A. Nodal explants cultured on MS medium supplemented with $2.5 \mathrm{mg} \cdot 1^{-1} \mathrm{TDZ}, 50 \mathrm{mg} \cdot 1^{-1} \mathrm{AdS}$ and $0.1 \mathrm{mg} \cdot 1^{-1} \mathrm{NAA}$ (showing hyperhydric shoots). B. Nodal explants cultured on MS medium supplemented with $2.5 \mathrm{mg} \cdot 1^{-1} \mathrm{mT}, 50 \mathrm{mg} \cdot \mathrm{l}^{-1} \mathrm{AdS}$ and $0.1 \mathrm{mg} \cdot 1^{-1} \mathrm{NAA}$ (showing healthy and uniform shoots). 


\begin{tabular}{|c|c|c|c|c|c|}
\hline 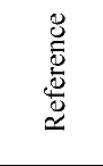 & 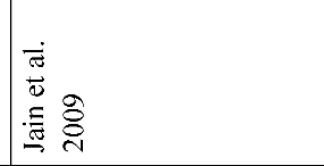 & 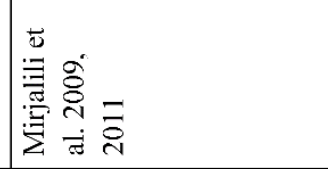 & 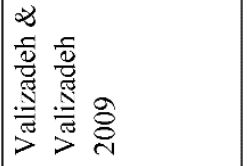 & 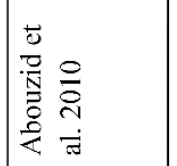 & 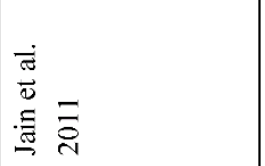 \\
\hline 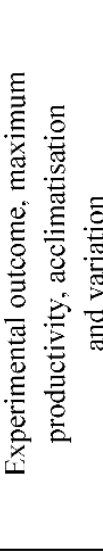 & 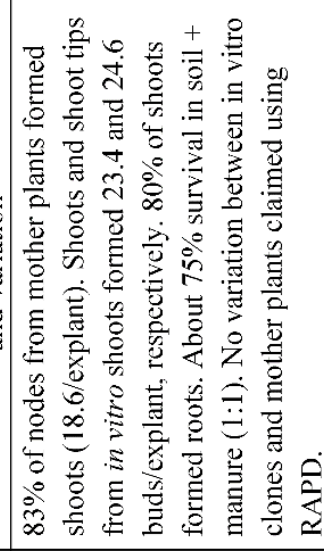 & 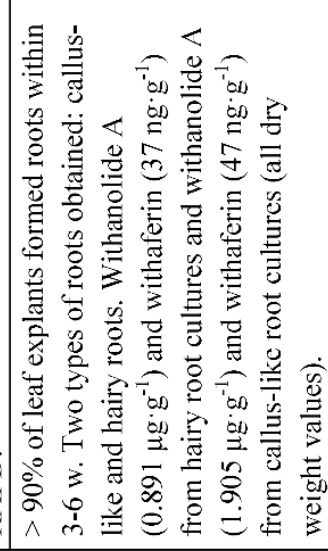 & 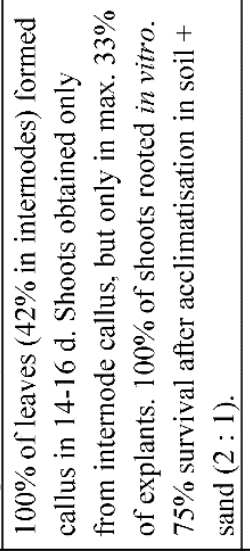 & 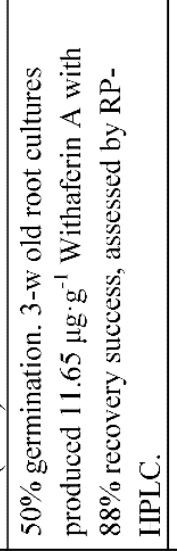 & 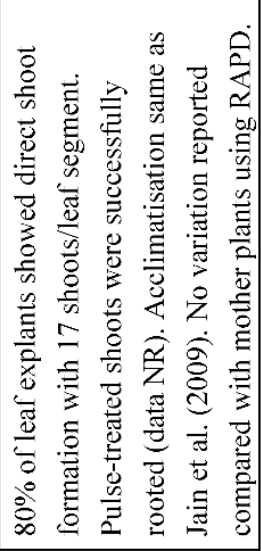 \\
\hline 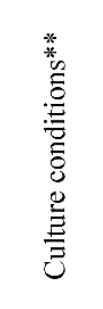 & 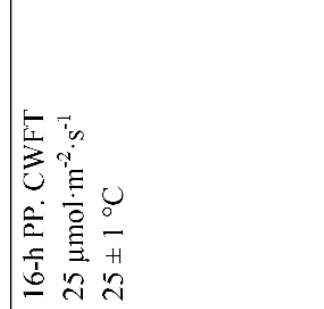 & 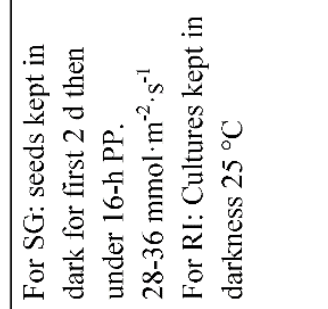 & 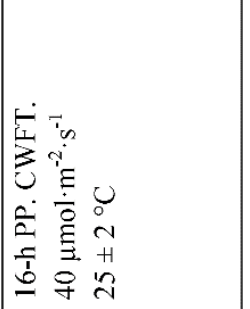 & 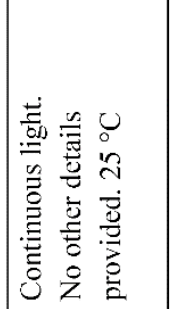 & 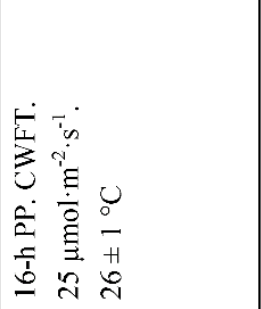 \\
\hline 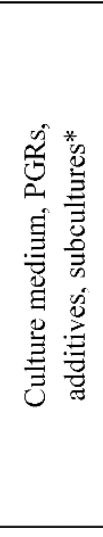 & 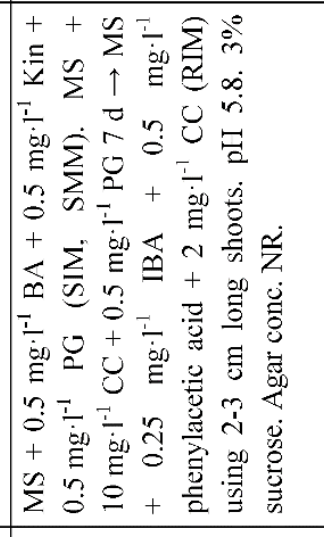 & 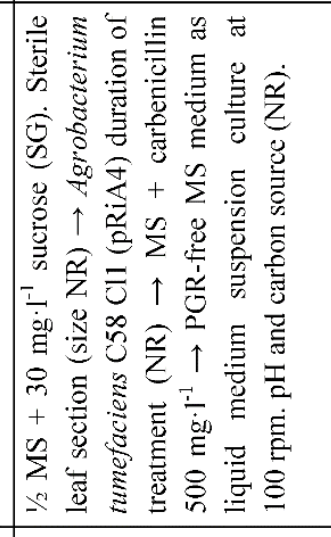 & 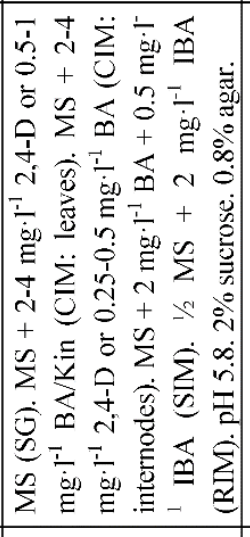 & 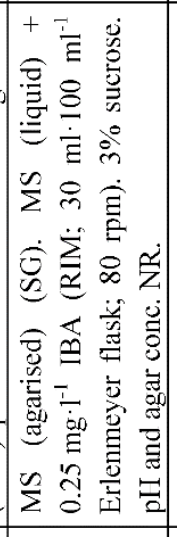 & 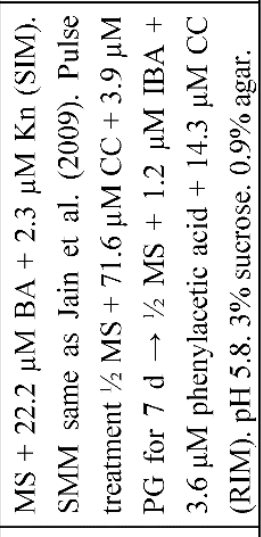 \\
\hline 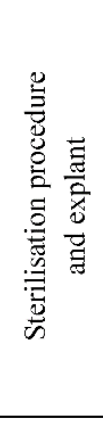 & 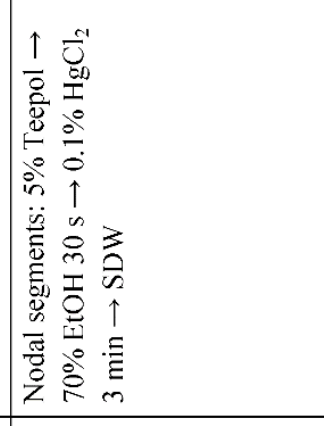 & 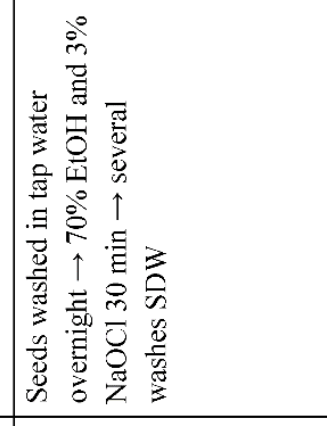 & 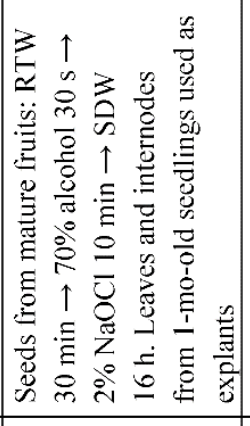 & 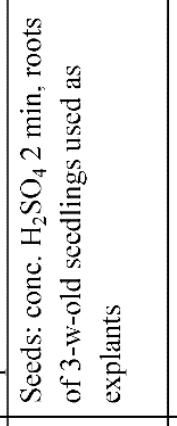 & 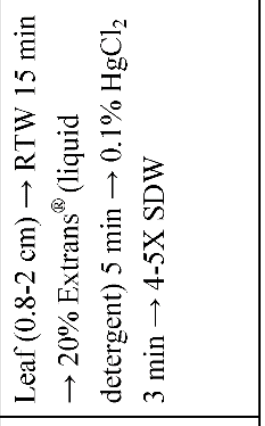 \\
\hline 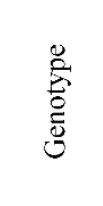 & 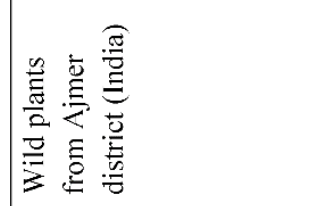 & 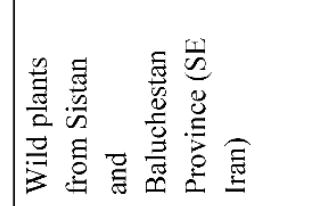 & 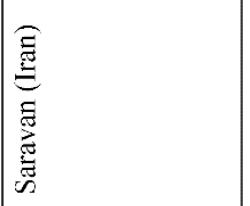 & 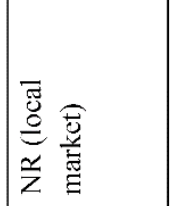 & 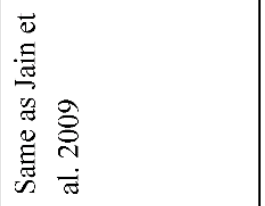 \\
\hline
\end{tabular}




\begin{tabular}{|c|c|c|c|c|}
\hline 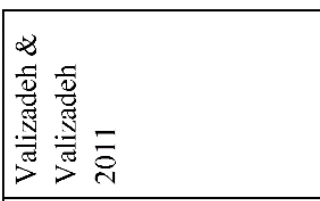 & 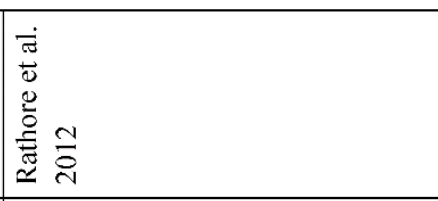 & 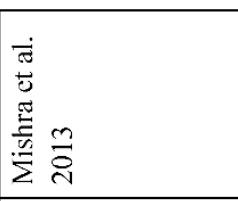 & 竞 & 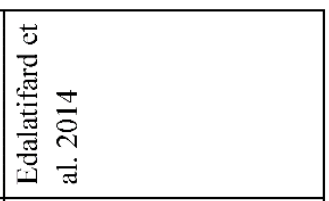 \\
\hline 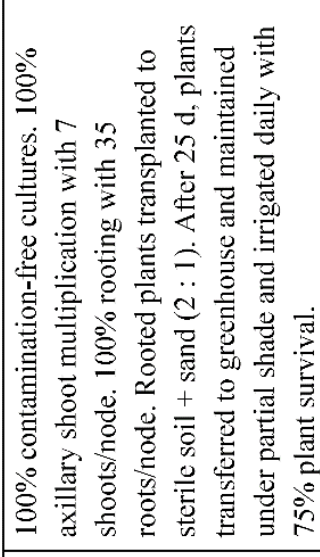 & 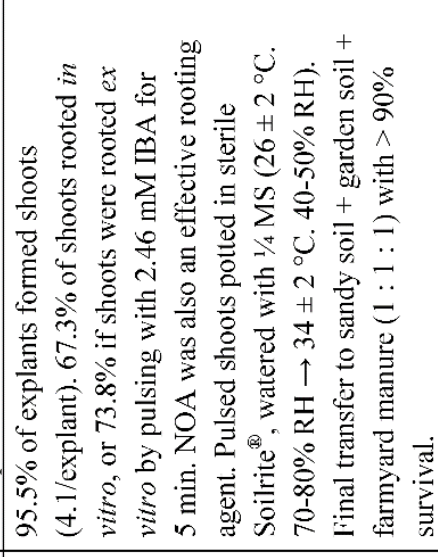 & 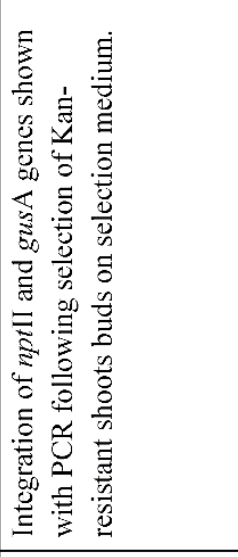 & 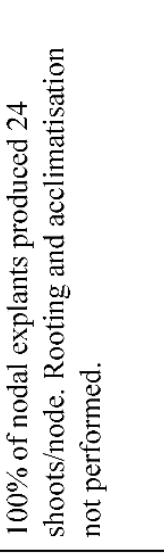 & 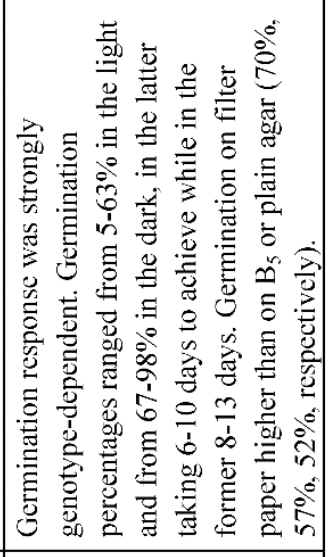 \\
\hline 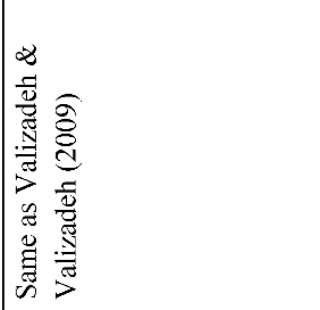 & 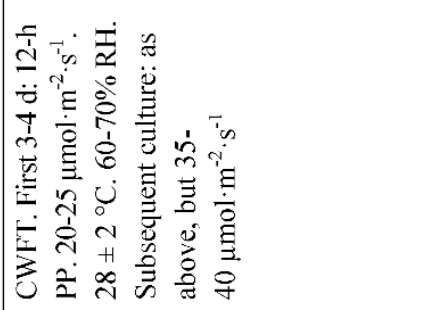 & 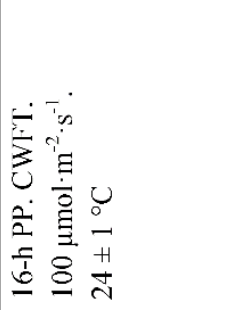 & 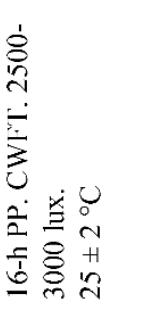 & 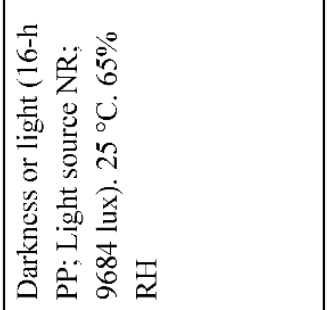 \\
\hline 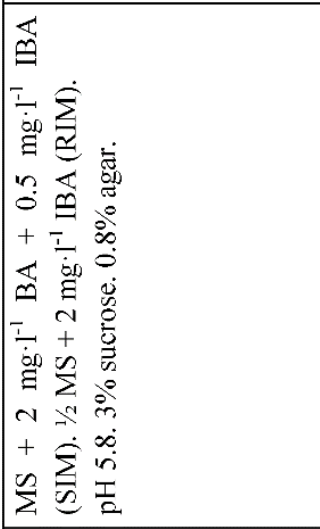 & 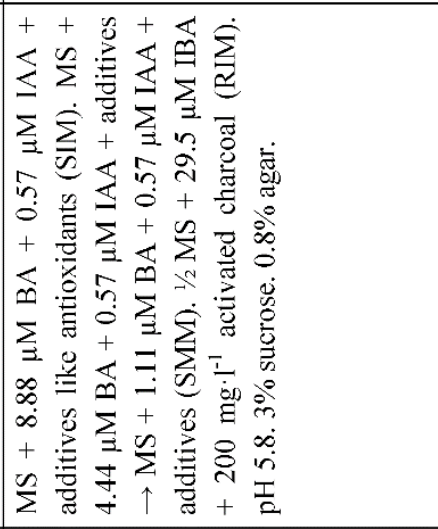 & 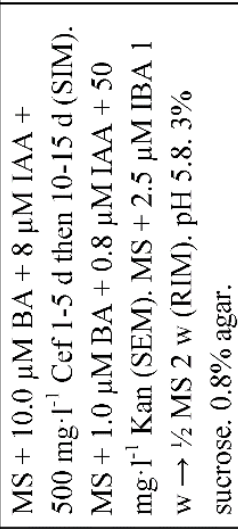 & 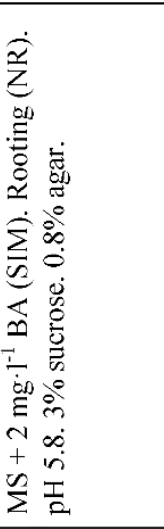 & 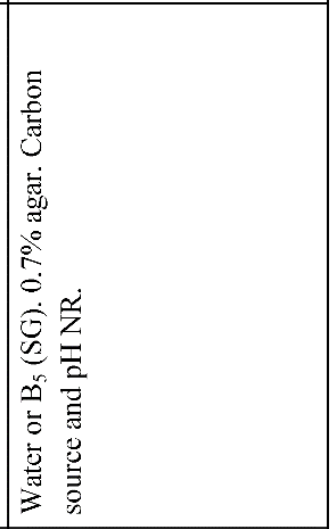 \\
\hline 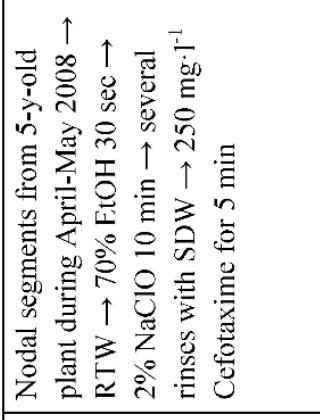 & 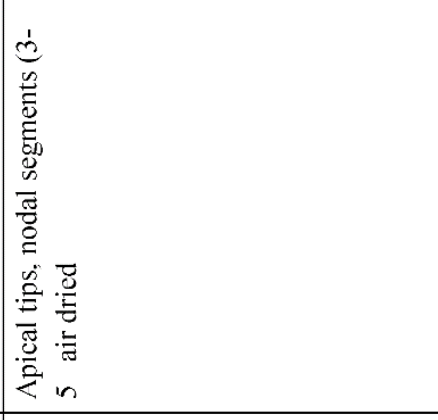 & 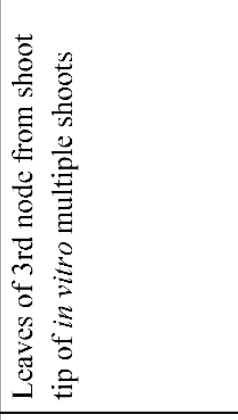 & 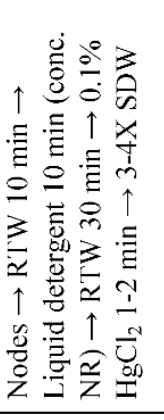 & 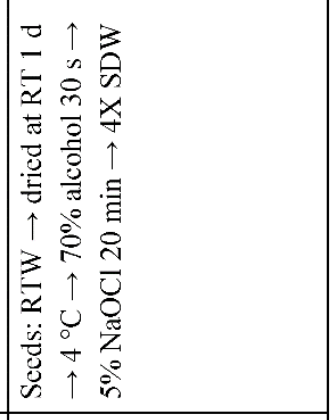 \\
\hline 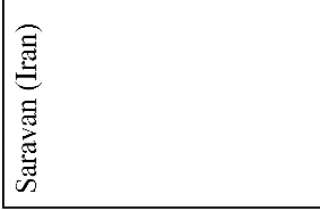 & 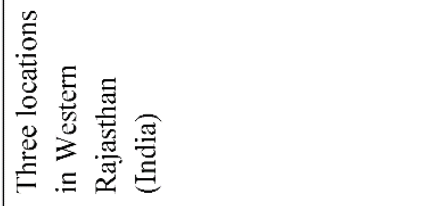 & $\frac{\tilde{z}}{\mathrm{z}}$ & 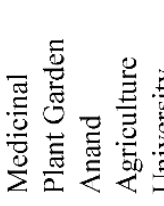 & 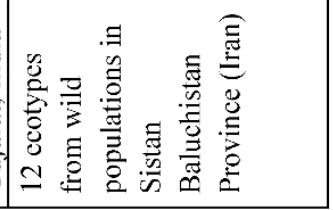 \\
\hline
\end{tabular}




\begin{tabular}{|c|c|c|}
\hline 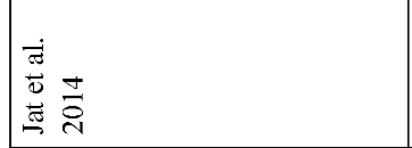 & 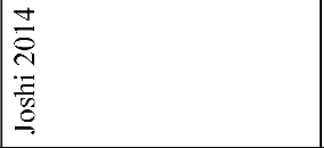 & 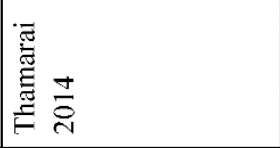 \\
\hline 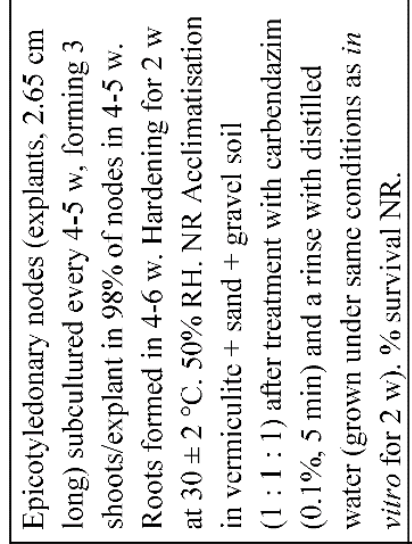 & 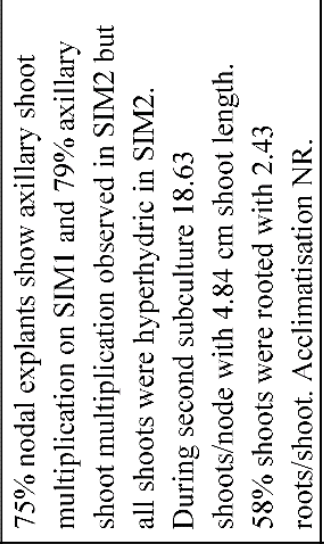 & 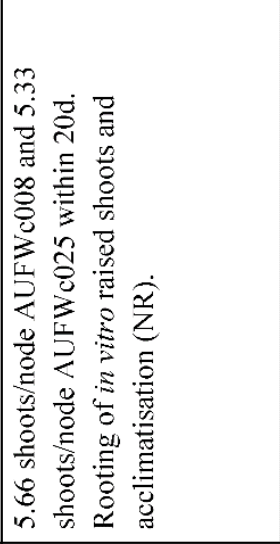 \\
\hline 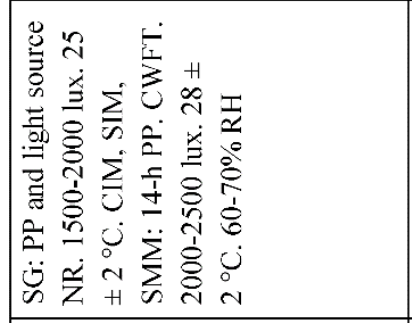 & 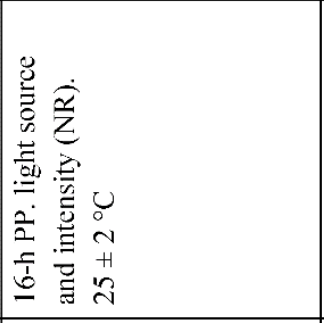 & 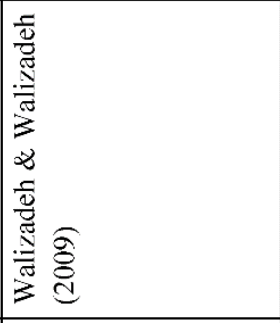 \\
\hline 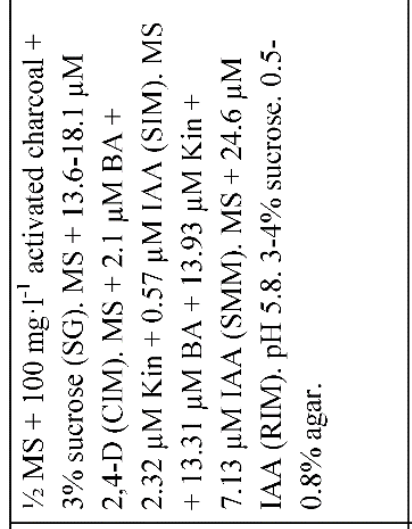 & 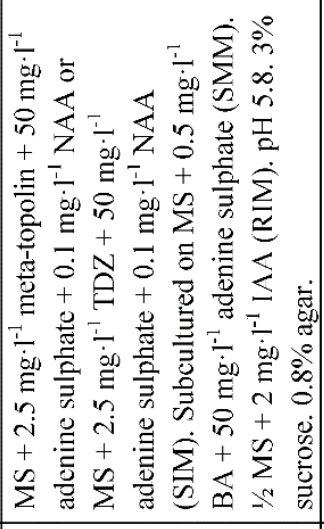 & 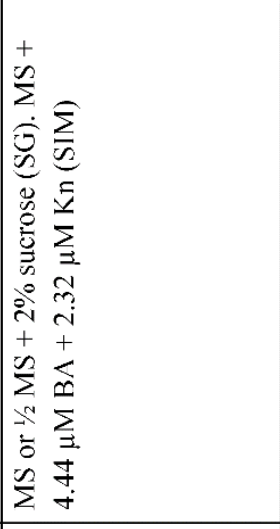 \\
\hline 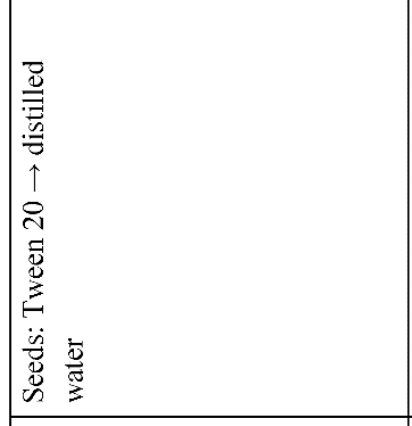 & 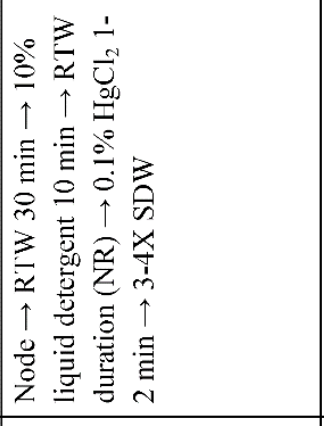 & 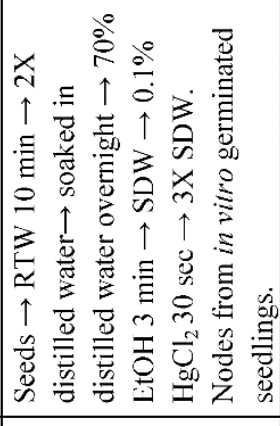 \\
\hline $\begin{array}{l}\frac{\tilde{g}}{\tilde{g}} \\
\vec{g}\end{array}$ & 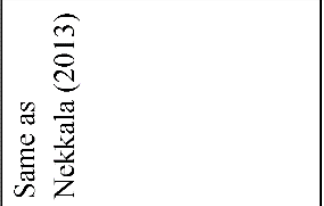 & 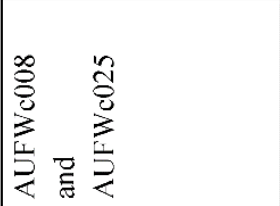 \\
\hline
\end{tabular}

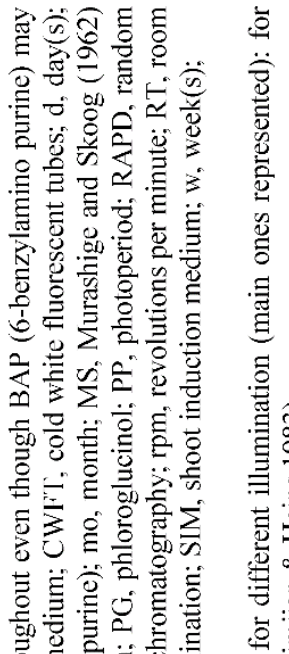

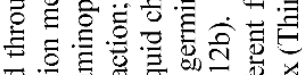

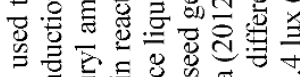

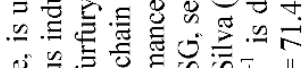

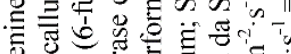

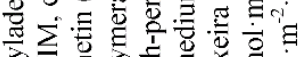

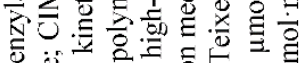

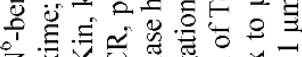

乙 \论

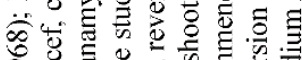

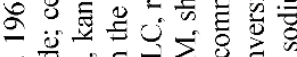

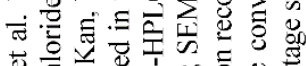

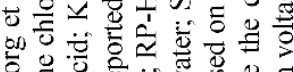

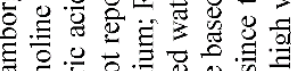

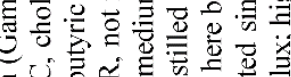

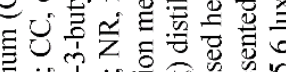

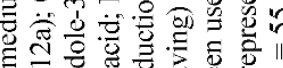
का

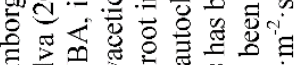

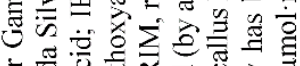

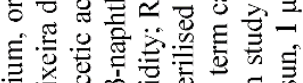

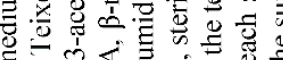
仓。 on

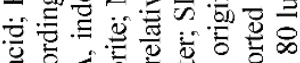

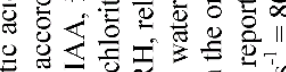

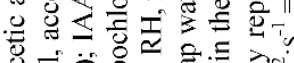

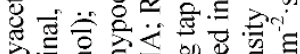

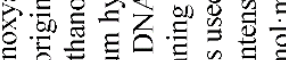

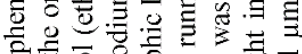

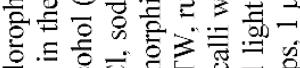
항

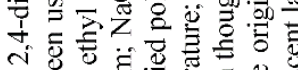
人ํ.

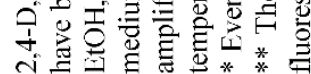


In order to produce withanolide $\mathrm{A}$ in root cultures as an alternative method to the destructive harvesting of roots, Abouzid et al. (2010) established liquid root cultures based on IBA-supplemented MS medium using seedling-derived roots as explants. One proposal for future experiments can be the use of exogenously applied PG in order to increase root production (Teixeira da Silva et al. 2013).

There are very limited studies that have employed molecular techniques to assess any aspect of W. coagulans research. For example, Jain et al. $(2009,2011)$ used random amplified polymorphic DNA to assess deviations from true-to-type plantlets derived from in vitro culture and mother plants. Kushwaha et al. (2013) characterized a tropinone reductase in $W$. coagulans using leaves from in vitro plantlets according to protocols established by Jain et al. (2009, 2011) and Mishra et al. (2013). The study by Mishra et al. (2013) was the first study on an enzyme involved in the secondary metabolism in this medicinal plant. Mirjalili et al. (2011) capitalized upon the ability of hairy root cultures, induced by Agrobacterium rhizogenes-mediated transformation, to increase the production of triterpenoids (phytosterols and withanolides) through the overexpression of the squalene synthase gene. Mishra et al. (2013) established the first Agrobacterium tumefaciens-mediated genetic transformation protocol for $W$. coagulans in which leaves of in vitro plantlets were used to introduce marker (gusA) and selector (nptII, hptII) genes.

Synthetic seed technology, which involves the encapsulation of in vitro or in vivo generated explants in an alginate bead, is an efficient system that supports the multiplication, storage and exchange of germplasm having traits of choice that are difficult to propagate via traditional approaches (Sharma et al., 2013; Gantait et al., 2015). Rathore \& Kheni (2015) recently reported the alginate encapsulation of microcuttings (shoot tip and nodal segments) with $3.0 \%$ sodium alginate and $100 \mathrm{mM}$ calcium chloride. Microshoot encapsulates were regenerated (96\%) on $0.75 \%$ agar-gelled MS medium containing $1.11 \mu \mathrm{M}$ BAP and $0.57 \mu \mathrm{M}$ IAA. Pulse-treatment of the base of microcuttings with $2.46 \mathrm{mM}$ IBA for 2 min was essential for conversion of beads into plantlets, and more than $95 \%$ of the encapsulated microcuttings produced shoots and roots within $30 \mathrm{~d}$.

\section{Conclusion and future perspectives}

The tissue culture and biotechnology of W. coagulans, a valuable medicinal plant, remain unexplored relative to the more famous W. somnifera. The focus of future studies to improve the research objectives of this plant species is: induction of somatic embryogenesis and use of somatic embryos in bioreactors, the use of somatic embryos and other propagules, increased production of withanolide or withaferin, the most important biologically active compounds of this plant; the induction of organs using a wide range of plant growth regulators (e.g. Teixeira da Silva 2014; Teixeira da Silva et al. 2014) and testing the use of different gelling agents, abiotic growth conditions, additives and even biotic interactions (e.g. arbuscular mycorrhizal fungi) in order to improve in vitro productivity and secondary metabolite production. For the better understanding of developmental regulatory events, investigation of thin cell layers could be useful (Teixeira da Silva 2014; Teixeira da Silva et al. 2014) while in vitro breeding could advance much more quickly if flowers could be artificially induced in vitro (Teixeira da Silva 2014; Teixeira da Silva et al. 2014, 2015), allowing for the reproductive tissue to be available on demand throughout the year. The biotechnology of this plant must be accompanied with an increased environmental regulation that suppresses illegal gathering and destructive exploitation of this plant species from nature, while also seeking ways to introduce genetically variable in vitro-derived clones back into wild, protected environments.

\section{REFERENCES}

Abouzid S.F., El-Bassuony A.A., Nasib A., Khan S., Qureshi J., Choudhary M.I. 2010. Withaferin a production by root cultures of Withania coagulans. International Journal of Applied Research in Natural Products 3: 23-27.

Edalatifard L., Modarres-Sanavy S.A.M., Askari H. 2014. The optimum condition under light and media for seed germination of Withania coagulans. International Journal of Farming and Allied Sciences 3: $722-728$. 
Gamborg O.L., Miller R.A., Ojima K. 1968. Nutrient requirements of suspension cultures of soybean root cells. Experimental Cell Research 50: 151-158. DOI: 10.1016/0014-4827(68)90403-5.

Gantait, S., Kundu, S., Ali, N., Sahu, N.C., 2015. Synthetic seed production of medicinal plants: a review on influence of explants, encapsulation agent and matrix. Acta Physiologiae Plantarum 37: 98. DOI: 10.1007/s11738-015-1847-2.

Gilani S.A., Kikuchi A., Watanabe K.N. 2009. Genetic variation within and among fragmented populations of endangered medicinal plant, Withania coagulans (Solanaceae) from Pakistan and its implications for conservation. African Journal of Biotechnology 8: 2948-2958. DOI: 10.5897/AJB09.525.

Gupta P.C. 2012. Withania coagulans Dunal - an overview. International Journal of Pharmaceutical Sciences Review and Research 12: 68-71.

Gupta V., Keshari B.B. 2013. Withania coagulans Dunal (Paneer Doda): a review. International Journal of Ayurvedic and Herbal Medicine 3(5): 1130-1136.

Jain R., Kachhwaha S., Kothari S.L. 2012. Phytochemistry, pharmacology, and biotechnology of Withania somnifera and Withania coagulans: a review. Journal of Medicinal Plants Research 6: 5388-5399. DOI: $10.5897 / J M P R 12.704$.

Jain R., Sinha A., Jain D., Kachhwaha S., Kothari S.L. 2011. Adventitious shoot regeneration and in vitro biosynthesis of steroidal lactones in Withania coagulans (Stocks) Dunal. Plant Cell Tissue and Organ Culture 105: 135-140. DOI: 10.1007/s11240-0109840-3.

Jain R., Sinha A., Kachhwaha S., Kothari S.L. 2009. Micropropagation of Withania coagulans (Stocks) Dunal: A critically endangered medicinal herb. Journal of Plant Biochemistry and Biotechnology 18: 249-252. DOI: 10.1007/bf03263330.

Jat B.L., Meena G.P., Choudhary C.R., Maheshwari R.K., Jeswani G. 2014. In vitro propagation of Withania coagulance [sic] through seedling segment (epicotyledonary node). International Journal of Chemistry and Pharmaceutical Sciences 2: 979-989.

Joshi H. 2014. Tissue culture studies on Withania coagulans and Punica granatum cv. Bhagava. Industrial Biotechnology M.Sc. Thesis. Sardar Patel University.

Mirjalili M.H., Bonfill M., Moyano E., Cusido R.M., Palazón J. 2009. Overexpression of the Arabidopsis thaliana squalene synthase gene in Withania coagulans hairy root cultures increases the biosynthesis of phytosterols and withanolides. New Biotechnology 25: S334. DOI: 10.1016/j.nbt.2009.06.809.
Mirjalili M.H., Moyano E., Bonfill M., Cusido R.M., Palazón J. 2011. Overexpression of the Arabidopsis thaliana squalene synthase gene in Withania coagulans hairy root cultures. Biologia Plantarum 55: 357-360. DOI: 10.1007/s10535-011-0054-2.

Mishra S., Sangwan R.S., Bansal S., Sangwan N.S. 2013. Efficient genetic transformation of Withania coagulans (Stocks) Dunal mediated by Agrobacterium tumefaciens from leaf explants of in vitro multiple shoot culture. Protoplasma 250: 451-458. DOI: 10.1007/s00709-012-0428-0.

Murashige T., Skoog F. 1962. A revised medium for rapid growth and bioassays with tobacco tissue cultures. Physiologia Plantarum 15: 473-497. DOI: 10.1111/j.1399-3054.1962.tb08052.x.

Nekkala S.K. 2013. Effect of cytokinins and auxins and various gelling agents in in vitro shoot proliferation of Withania coagulans (Stocks) Dunal. Biochemistry M.Sc. Thesis. Sardar Patel University.

Panwar J., Tarafdar J.C. 2006. Distribution of three endangered medicinal plant species and their colonization with arbuscular mycorrhizal fungi. Journal of Arid Environments 65: 337-350. DOI: 10.1016/j.jaridenv.2005.07.008.

Rathore M.S., Shekhawat S., Kaur G., Singh R.P., Shekhawat N.S. 2012. Micropropagation of vegetable rennet (Withania coagulans [Stocks] Dunal) a critically endangered medicinal plant. Journal of Sustainable Forestry 31: 727-746. DOI: 10.1080/10549811.2012.706533.

Rathore, M.S., Kheni, J., 2015. Alginate encapsulation and in vitro plantlet regeneration in critically endangered medicinal plant, Withania coagulans (Stocks) Dunal. Proceedings of the National Academy of Sciences, India Section B: Biological Sciences. DOI: 10.1007/s40011-015-0577-y

Sharma S., Shahzad A., Teixeira da Silva J.A. 2013. Synseed technology - A complete synthesis. Biotechnology Advances 31(2): 186-207. DOI: 10.1016/j.biotechadv.2012.09.007.

Teixeira da Silva J.A., Dobránszki J., Ross S. 2013. Phloroglucinol in plant tissue culture. In Vitro Cellular \& Developmental Biology - Plant 49: 1-16. DOI: 10.1007/s11627-013-9491-2.

Teixeira da Silva J.A. 2012a. Is BA (6-benzyladenine) BAP (6-benzylaminopurine)? The Asian and Australasian Journal of Plant Science and Biotechnology 6: 121-124.

Teixeira da Silva J.A. 2012b. Callus, calluses or calli: multiple plurals? The Asian and Australasian Journal of Plant Science and Biotechnology 6: 125-126. 
Teixeira da Silva J.A. 2014. Response of hybrid Cymbidium (Orchidaceae) protocorm-like bodies to 26 plant growth regulators. Botanica Lithuanica 20: 3-13. DOI: 10.2478/botlit-2014-0001.

Teixeira da Silva J.A., Kerbauy G.B., Zeng S., Chen Z., Duan J. 2014. In vitro flowering of orchids. Critical Reviews in Biotechnology 34: 56-76. DOI: 10.3109/07388551.2013.807219.

Teixeira da Silva J.A., Lema-Rumińska J., Tymoszuk A., Kulpa D. 2015. Regeneration from Chrysanthemum flowers: a review. Acta Physiologiae Plantarum 37: 36. DOI: 10.1007/s11738-015-1773-3.

Thamarai R.S. 2014. Comparative evaluation of in vitro growth characteristic and secondary metabolite accumulation in two cultivars of Withania coagulans
AUWc 008 and AUWc 025. Biotechnology M.Sc. Thesis, Sardar Avinashilingam Institute for Home Science and Higher Education.

Thimijan R.W., Heins R.D. 1983. Photometric, radiometric, and quantum light units of measure: a review of procedures for interconversion. HortScience 18: 818-822.

Valizadeh J., Valizadeh M. 2009. In vitro callus induction and plant regeneration from Withania coagulans: a valuable medicinal plant. Pakistan Journal of Biological Sciences 12: 1415-1419. DOI: 10.3923/pjbs.2009.1415.1419.

Valizadeh J., Valizadeh M. 2011. Development of efficient micropropagation protocol for Withania coagulans (Stocks) Dunal. African Journal of Biotechnology 10: 7611-7616. DOI: 10.5897/AJB11.075. 